\title{
ON TWO LONG STANDING OPEN PROBLEMS ON $L_{p}$-SPACES
}

\begin{abstract}
POPOV M.M. ${ }^{1,2}$
The present note was written during the preparation of the talk at the International Conference dedicated to 70-th anniversary of Professor O. Lopushansky, September 16-19, 2019, IvanoFrankivsk, Ukraine. We focus on two long standing open problems. The first one, due to Lindenstrauss and Rosenthal (1969), asks of whether every complemented infinite dimensional subspace of $L_{1}$ is isomorphic to either $L_{1}$ or $\ell_{1}$. The second problem was posed by Enflo and Rosenthal in 1973: does there exist a nonseparable space $L_{p}(\mu)$ with finite atomless $\mu$ and $1<p<\infty, p \neq 2$, having an unconditional basis? We analyze partial results and discuss on some natural ideas to solve these problems.
\end{abstract}

Key words and phrases: $L_{p}$-spaces, complemented subspace, unconditional basis.

\footnotetext{
${ }^{1}$ Vasyl Stefanyk Precarpathian National University, 57 Shevchenka str., 76018, Ivano-Frankivsk, Ukraine

2 Pomeranian University in Słupsk, 76-200, Słupsk, Poland

E-mail: misham.popovegmail.com
}

\section{INTRODUCTION}

Investigation of the geometry of Lebesgue spaces $L_{p}:=L_{p}[0,1]$ has long and rich history (see [3]) due to famous mathematicians: D.E. Alspach, S. Banach, J. Bourgain, D.L. Burkholder, L.E. Dor, P. Enflo, W.B. Johnson, M.I. Kadets, N. Kalton, J. Lindenstrauss, B. Maurey, E. Odell, R.E.A.C. Paley, A. Pełczyński, H.P. Rosenthal, G. Schechtman, T.W. Starbird, S. Szarek, M. Talagrand, L. Tzafriri and others. More is known on the isomorphic structure of these classical spaces. Isomorphic embeddability of $L_{r}(v)$ into $L_{p}$ is completely known. We use the notation $X \hookrightarrow Y$ to express that $X$ embeds isomorphically into $Y$, and $X \simeq Y$ means that the Banach spaces $X$ and $Y$ are isomorphic. The relation $\ell_{p} \hookrightarrow L_{p}$, which is easily seen, was first noted by S. Banach [4, p. 175]. The embedding $\ell_{2} \hookrightarrow L_{p}$ follows from Khintchin's inequality [30, p. 66]. It is not hard to see that $\ell_{p} \nrightarrow L_{2}$ for $p \neq 2$ (for the proof, see [4, p. 175]). The relation $\ell_{r} \leftrightarrow L_{p}$ for $2<p<r$ and $1 \leq r<p<2$ was proved by S. Banach [4, p. 175]. Paley's results [37] imply $\ell_{r} \nrightarrow L_{p}$ for $1 \leq r<2<p, 2<r<p$ and $1 \leq p<2<r$.

A special case is $1 \leq p<r<2$, where isometric embeddings of $L_{r}$ into $L_{p}$ are possible. First it was proved by P. Levy [25] that $\ell_{r}$ is finitely representable ${ }^{1}$ in $L_{p}$ if $1 \leq p<r<2$. Later M.I. Kadets proved that $\ell_{r} \hookrightarrow L_{p}$ for $1 \leq p<r<2$ [20]. Then the latter result was strengthen to the embedding $L_{r} \hookrightarrow L_{p}$ by J. Bretagnolle, D. Dacunha-Castelle and J. L. Krivine [9] and independently by J. Lindenstrauss J. and A. Pełczyński [27], who proved more: if a Banach space $X$ is finitely representable in $L_{p}$ then $X \hookrightarrow L_{p}$.

$\mathrm{y} \triangle \mathrm{K} 517.982$

2010 Mathematics Subject Classification: Primary 46B03; secondary 46B15, 46B26.

${ }^{1}$ A Banach space $X$ is said to be finitely representable in a Banach space $Y$ if for every $\varepsilon>0$ and every finite dimensional subspace $F$ of $X$ there exists a subspace $G$ of $Y$ of the same dimension such that $d(F, G)<1+\varepsilon$, where $d(F, G)$ denotes the Banach-Mazur distance between $F$ and $G$. 
As we see, the properties of the spaces $L_{p}$ are different for the cases $p<2$ and $p>2$. Moreover, if $2<p<\infty$ then every subspace of $L_{p}$ possesses the following properties:

- either is isomorphic to a Hilbert space or contains a complemented subspace isomorphic to $\ell_{p}[21]$

- either contains a subspace isomorphic to $\ell_{2}$ or embeds isomorphically into $\ell_{p}$ [19].

On the other hand, if $1 \leq p<2$ then every subspace of $L_{p}$ either contains a complemented subspace isomorphic to $\ell_{p}$ or embeds isomorphically into $L_{r}$ for some $p<r \leq 2$ [44].

Acknowledgments. The author is grateful to W. B. Johnson and G. Schechtman for valuable remarks.

\section{COMPLEMENTEd SUbSPACES OF $L_{p}$}

\subsection{As $p$ goes to 1, the complementability properties of subspaces of $L_{p}, p \neq 2$, get worse}

By Khintchin's inequality, the closed linear span $R$ of the Rademacher system in $L_{p}$ is isomorphic to $\ell_{2}$ and is actually independent on $p$, as a set. Remark that $R$ is complemented in $L_{p}$ for $1<p<\infty$ [30, p. 66] and is uncomplemented in $L_{1}$, as well as any other subspace of $L_{1}$ isomorphic to $\ell_{2}$ [38]. So, it became interesting, whether there exists an uncomplemented subspace of $L_{p}$ isomorphic to $\ell_{2}$ for $p>1$. If $2<p<\infty$ then every subspace $X$ of $L_{p}$ isomorphic to $\ell_{2}$ is complemented, and, moreover, the $L_{p^{-}}$and $L_{2}$-norms ${ }^{2}$ on $X$ are equivalent [21]. To the contrast, if $1<p<2$ then there exists an uncomplemented subspace of $L_{p}$ isomorphic to $\ell_{2}$ (first it was proved for $1<p<4 / 3$ in [42] and then for the rest of values in [5]).

It is clear that $L_{p}$ contains a complemented subspace isomorphic to $\ell_{p}$. If $1 \leq p<\infty, p \neq 2$, then there is an uncomplemented subspace of $L_{p}$ isomorphic to $\ell_{p}$, and hence, it is not difficult to show that there is an uncomplemented subspace of $L_{p}$ isomorphic to $L_{p}$ itself (first it was proved for $2<p<\infty$ and $1<p<4 / 3$ in [43], then in a different way for all $1<p<2$ in [5], and finally for $p=1$ in [6]).

\subsection{Primarity of $L_{p}$ and Enflo operators}

By the famous Enflo theorem, if $L_{p}=X \oplus Y, 1 \leq p<\infty$, is a decomposition into mutually complemented subspaces, then at least one of the subspaces $X, Y$ is isomorphic to $L_{p}$ (first it was announced by P. Enflo; then B. Maurey [34] published a proof, see also [2] for all $p$, [14] for $p=1$ and [31, p. 179] for a generalization to rearrangement invariant spaces). This nice property of the spaces $L_{p}$ is called the primarity.

Let $X, Y$ be Banach spaces. Denote by $\mathcal{L}(X, Y)$ the Banach space of all continuous linear operators from $X$ to $Y$, and write $\mathcal{L}(X)$ instead of $\mathcal{L}(X, X)$. Recall that an operator $T \in \mathcal{L}(X, Y)$ is said to fix a copy of a Banach space $Z$, if there exists a subspace $X_{1}$ of $X$ isomorphic to $Z$ such that the restriction $\left.T\right|_{X_{1}}$ of $T$ to $X_{1}$ is an into isomorphism. An operator $T \in \mathcal{L}\left(L_{p}, Y\right)$, $1 \leq p<\infty$, is called an Enflo operator provided $T$ fixes a copy of $L_{p}$. Note that every Enflo operator $T \in \mathcal{L}\left(L_{p}\right)$ fixes a complemented copy of $L_{p}$, that is, there is a complemented subspace $X_{1}$ of $X$ isomorphic to $L_{p}$ such that the restriction $\left.T\right|_{X_{1}}$ is an into isomorphism, because every subspace $X$ of $L_{p}$, which is isomorphic to $L_{p}$, contains a further subspace $Y \subseteq X$ isomorphic to

\footnotetext{
${ }^{2}$ which are well defined for these values of $p$
} 
$L_{p}$ and complemented in $L_{p}$ (see [18, p. 239] for $p>1$ and [14] for $p=1$ ). The Enflo theorem implies that, if the identity operator $I d$ on $L_{p}$ is a sum of two projections $I d=P+Q$, then at least one of the projections $P, Q$ is an Enflo operator. Moreover, the range of a projection $P$ on $L_{p}$ is isomorphic to $L_{p}$ if and only if $P$ is an Enflo operator (to prove, use the mentioned above result from [18] and Pełczyński's decomposition method [31, p. 54]).

\subsection{Isomorphic types of complemented subspaces of $L_{p}$}

How many do there exist pairwise non-isomorphic complemented subspaces of $L_{p}$ for $1 \leq p<\infty, p \neq 2$ ? If $p>1$ then there are obviously the following pairwise non-isomorphic Banach spaces isomorphic to complemented subspaces of $L_{p}$ :

$$
L_{p}, \quad \ell_{p}, \quad \ell_{2}, \quad \ell_{p} \oplus \ell_{2}, \quad\left(\bigoplus_{n=1}^{\infty} \ell_{2}\right)_{p} .
$$

Further finitely many examples, different from the above obvious ones, was obtained by H.P. Rosenthal in [43]. Later G. Schechtman provided infinitely many pairwise non-isomorphic examples in [48], and then J. Bourgain, H.P. Rosenthal and G. Schechtman constructed uncountably many pairwise non-isomorphic complemented subspaces of $L_{p}$ for $1<p<\infty$, $p \neq 2$ in [8] (it is unknown, whether there exists continuum such subspaces).

The exceptional case is $p=1$ : there are only two known obvious examples of pairwise non-isomorphic infinite dimensional subspaces of $L_{1}$, they are $L_{1}$ itself and $\ell_{1}$.

Problem 1 (Lindenstrauss and Rosenthal, 1969, [29]). Is every complemented infinite dimensional subspace of $L_{1}$ isomorphic to either $L_{1}$ or $\ell_{1}$ ?

\subsection{Progress in the solution of Problem 1}

The following assertions have been established for an arbitrary complemented subspace $E$ of $L_{1}$.

Theorem 1 (Pełczyński, 1960, [38]). E contains a subspace isomorphic to $\ell_{1}$ and complemented in $L_{1}$.

Theorem 2 (Lindenstrauss, Pełczyński, 1968, [27]). If $E$ has an unconditional basis then $E$ is isomorphic to $\ell_{1}$.

Recall that the Radon-Nikodym property (RNP) for a Banach space $X$ means that for every finite measure space $(\Omega, \Sigma, \mu)$ and every $\mu$-continuous $X$-valued measure $G: \Sigma \rightarrow X$ of bounded variation there exists $g \in L_{1}(\mu, X)$ such that $G(A)=\int_{A} g d \mu$ for all $A \in \Sigma$. One can show that the characteristic function $G(A)=\mathbf{1}_{A}$ is an example of $L_{1}$-valued such measure for which the function $g$ does not exist [12, p. 61]; thus, $L_{1}$ does not have the RNP. However, $\ell_{1}$ has the RNP (this can be proved directly, using the Radon-Nikodým theorem for separate coordinates $[12$, p. 64]).

Theorem 3 (Lewis, Stegall, 1973, [26]). If $E$ has the RNP then $E$ is isomorphic to $\ell_{1}$.

A Banach space $X$ is said to have the Schur property if the weak convergence of a sequence in $X$ implies its norm convergence. It is well known that $\ell_{1}$ has the Schur property. 
Theorem 4 (Rosenthal, 1975, [45]). If $E$ does not have the Schur property then $\ell_{2}$ embeds into E.

Theorem 5 (Enflo, Starbird, 1979, [14]). If E contains a subspace isomorphic to $L_{1}$ then $E$ is itself isomorphic to $L_{1}$.

Simultaneously, W.B. Johnson, B. Maurey, G. Schechtman and L. Tzafriri [18] obtained the same result as Theorem 5 asserts for $L_{p}$ with $1<p<\infty$.

The next result strengthens Theorem 4.

Theorem 6 (Bourgain, 1980, [7]). If E does not have the Schur property then $\left(\bigoplus_{n=1}^{\infty} \ell_{2}\right)_{1}$ embeds into $E$.

There is a natural idea to solve Problem 1. Obviously, the hypothesis that every complemented infinite dimensional subspace of $L_{1}$ is isomorphic to either $L_{1}$ or $\ell_{1}$, is equivalent to the hypothesis that the following two climes hold true.

Let $E$ be an infinite dimensional complemented subspace of $L_{1}$.

Claim 1. If $E$ has the Schur property then $E$ is isomorphic to $\ell_{1}$.

Claim 2. If $E$ does not have the Schur property then $E$ is isomorphic to $L_{1}$.

As to the best of our knowledge, there is no information about Claim 1 in the literature. Remark that there is no direct way to prove Claim 1 without taking into account peculiarity of $L_{1}$, because there exists a Banach space with the Schur property but without the RNP, and so, not isomorphic to $\ell_{1}$ (see J. Hagler [17]).

However, Claim 2 has been considered by different mathematicians as a weak version of Problem 1 in the sense that a positive solution to Problem 1 implies a positive answer to Problem 2.

Problem 2 ([45], [14] and [7]). Must a non-Dunford-Pettis projection $P \in \mathcal{L}\left(L_{1}\right)$ be an Enflo operator? Equivalently, whether each non-Schur complemented subspace of $L_{1}$ is isomorphic to $L_{1}$ ?

The most unclear thing concerning Problem 2 is how to use the information that $P$ is a projection, not just a continuous linear operator. H.P. Rosenthal constructed an example of a non-Dunford-Pettis operator $T \in \mathcal{L}\left(L_{1}\right)$ failing to be an Enflo operator [45]. This is the socalled biased coin convolution operator. To explain the details, recall that the Rademacher system is defined by $r_{n}(t)=\operatorname{sign} \sin \left(2^{n+1} \pi t\right)$ for each $n \in \mathbb{N}$ and $t \in[0,1]$. Denote by $\mathbb{N}^{<\omega}$ the set of all finite subsets of $\mathbb{N}$. The Walsh system $\left(w_{I}\right)_{I \in \mathbb{N}<\omega}$ is defined by setting $w_{I}=\prod_{i \in I} r_{i}$, where $\left(r_{n}\right)_{n=1}^{\infty}$ is the Rademacher system (in particular, $w_{\varnothing}=\mathbf{1}$, by convention). The Walsh system with respect to the lexicographical order $w_{\varnothing}, w_{\{1\}}, w_{\{2\}}, w_{\{1,2\}}, w_{\{3\}}, w_{\{1,3\}}, w_{\{2,3\}}, w_{\{1,2,3\}}, \ldots$ is a Schauder basis of $L_{p}$ for $1<p<\infty$, an orthonormal basis of $L_{2}$, a conditional basis of $L_{p}$ for $p \neq 2$, and a Markushevich basis of $L_{1}$.

Theorem 7 (H.P. Rosenthal, [45]). There is $\varepsilon_{0} \in(0,1)$ such that for every $\varepsilon \in\left(0, \varepsilon_{0}\right)$ there is an operator $R_{\varepsilon} \in \mathcal{L}\left(L_{1}\right)$ possessing the equality $R_{\varepsilon} w_{I}=\varepsilon^{|I|} w_{I}$ for all $I \in \mathbb{N}^{<\omega}$, where $|I|$ is the cardinality of $I$.

The operator $R_{\varepsilon}$ is called the $\varepsilon$-biased coin convolution operator. Since $R_{\varepsilon} r_{n}=\varepsilon r_{n}$ for all $n \in \mathbb{N}$, the operator $R_{\varepsilon}$ is not Dunford-Pettis. H.P. Rosenthal proved in [45] that $R_{\varepsilon}$ is not an Enflo operator. 


\subsection{All operators on $L_{1}$ are regular}

Recall some information. Let $E, F$ be vector lattices. An operator $T: E \rightarrow F$ is called positive if $T\left(E^{+}\right) \subseteq F^{+}$, and $T: E \rightarrow F$ is called regular if $T$ equals a difference of two positive operators. Obviously, every positive (and hence, every regular) operator $T: E \rightarrow F$ is order bounded, that is, $T$ sends order bounded subsets of $E$ to order bounded subsets of $F$. Two elements $x, y \in E$ are said to be disjoint (write $x \perp y$ ) if $|x| \wedge|y|=0$. The notation $x=\bigsqcup_{k=1}^{n} x_{k}$ means that $x=\sum_{k=1}^{n} x_{k}$ and $x_{i} \perp x_{j}$ for $i \neq j$.

It is an amazing and seldom used fact on operators on $L_{1}$ that all of them are regular [47, p. 232]. More precisely, every operator $T \in \mathcal{L}\left(L_{1}\right)$ admits the representation $T=T^{+}-T^{-}$, where for every $x \in L_{1}^{+}$one has

$$
T^{+} x=\sup \left\{\sum_{k=1}^{m} T x_{k}: x=\bigsqcup_{k=1}^{n} x_{k}, n \in \mathbb{N}\right\} .
$$

As a consequence, we obtain that for any operator $T \in \mathcal{L}\left(L_{1}\right)$ the modulus $|T|=T^{+}+T^{-} \in$ $\mathcal{L}\left(L_{1}\right)$ exists and could be defined by setting for every $x \in E^{+}$

$$
|T| x=\sup \left\{\sum_{k=1}^{n}\left|T x_{i}\right|: x=\sum_{k=1}^{n} x_{k}, x_{k} \in E^{+}, n \in \mathbb{N}\right\} .
$$

Moreover, $\||T|\|=\|T\|$ for every $T \in \mathcal{L}\left(L_{1}\right)[47$, p. 232].

As was noted by H.P. Rosenthal [46], the regularity of operators $T \in \mathcal{L}\left(L_{1}(\mu), L_{1}(v)\right)$ is a consequence of the following Grothendieck's inequality [16, Corollaire, p. 67]: given any $f_{1}, \ldots, f_{1} \in L_{1}(\mu)$, one has

$$
\int_{\Omega_{\nu}} \max _{i}\left|T f_{i}\right| d \nu \leq\|T\| \int_{\Omega_{\mu}} \max _{i}\left|T f_{i}\right| d \mu .
$$

A very useful development of Grothendieck's inequality is M. Lévy's extension theorem (see [24]) asserting that, for every subspace $X$ of $L_{1}(\mu)$ every order bounded operator $T \in \mathcal{L}\left(X, L_{1}(v)\right)$ has an extension to some operator $\widehat{T} \in \mathcal{L}\left(L_{1}(\mu), L_{1}(v)\right)$, which is therefore order bounded as well. The latter fact was then generalized to regular operators from $L_{p}(\mu)$ to $L_{p}(v)$ for $1 \leq p \leq \infty$ by G. Pisier in [40].

The regularity of all operators on $L_{1}$ in fact means that there are few operators on $L_{1}$, only regular ones. This explains why common subspaces of all $L_{p}$ (like the closed linear span of the Rademacher system), which are complemented in $L_{p}$ for $p>1$ becomes uncomplemented in $L_{1}$ : they are complemented in $L_{p}$ by means of non-regular projections. The same reason makes the Haar system a conditional basis in $L_{1}$. This argument made the authors of [33] and [41, Problem 10.45] to generalize Problem 1 as follows. We say that a subspace $X$ of a Banach lattice is regularly complemented if there is a regular projection of $E$ onto $X$.

Problem 3. Let $1 \leq p<\infty, p \neq 2$. Is every regularly complemented subspace of $L_{p}$ isomorphic to either $\ell_{p}$ or $L_{p}$ ?

\subsection{Complemented subspaces of $L_{p}$ for $0<p<1$}

Consider now the quasi-Banach spaces $L_{p}$ for $0<p<1$. The list of known isomorphic types of complemented subspaces of these spaces becomes smaller by one space, namely by $\ell_{p}$, because $L_{p}$ has trivial dual and hence cannot have a complemented subspace with nontrivial dual, like those that are isomorphic to $\ell_{p}$. So, the problem is as follows. 
Problem 4. Let $0 \leq p<1$. Is every complemented subspace of $L_{p}$ isomorphic to $L_{p}$ ?

This problem has been systematically studied by N.J. Kalton in a number of papers. The best progress is Kalton's theorem, which asserts that, if there exists a complemented subspace of $L_{p}$ not isomorphic to $L_{p}$, then at most one, up to an isomorphism [22].

\section{UNCONDITIONAL BASES IN $L_{p}(\mu)$}

\subsection{Preliminary information}

For convenience of the reader, we recall some necessary information on bases $[1,30]$. A sequence $\left(x_{n}\right)_{n=1}^{\infty}$ of elements of a Banach space $X$ is called a Schauder basis (or just a basis) of $X$ if for every $x \in X$ there is a unique sequence of scalars $\left(a_{n}\right)_{n=1}^{\infty}$ such that

$$
x=\sum_{k=1}^{\infty} a_{k} x_{k}
$$

A sequence in $X$, which is a basis in its closed linear span, is called a basic sequence. The partial sums $P_{n} x=\sum_{k=1}^{n} a_{k} x_{k}$ of the expansion (1) are linear bounded projections on $X$ with $K:=\sup _{n}\left\|P_{n}\right\|<\infty$, and the number $K$ is called the basis constant of $\left(x_{n}\right)_{n=1}^{\infty}$. In particular, the coefficients $x_{k}^{*}(x):=a_{k}$ of the expansion (1) are elements of $X^{*}$ with $\sup _{n}\left\|x_{n}\right\|\left\|x_{n}^{*}\right\| \leq 2 \mathrm{~K}$ and are called the biorthogonal functionals to $\left(x_{n}\right)_{n=1}^{\infty}$. The best possible basis constant is 1 ; a basis with basis constant 1 is said to be monotone. The biorthogonal functionals $\left(x_{n}^{*}\right)_{n=1}^{\infty}$ form a basic sequence in $X^{*}$ with the same basis constant $K$. A basis $\left(x_{n}\right)_{n=1}^{\infty}$ of $X$ is called unconditional if for every $x \in X$ the series $x=\sum_{k=1}^{\infty} x_{k}^{*}(x) x_{k}$ converges unconditionally; otherwise the basis is said to be conditional. If $\left(x_{n}\right)_{n=1}^{\infty}$ is unconditional then for every sequence of signs $\Theta=\left(\theta_{n}\right)_{n=1}^{\infty}$, $\theta_{n} \in\{-1,1\}$, and every $x \in X$ the series $T_{\Theta} x:=\sum_{n=1}^{\infty} \theta_{n} x_{n}^{*}(x) x_{n}$ converges and $T_{\Theta}$ is a linear bounded operator. Moreover, $M:=\sup _{\Theta}\left\|T_{\Theta}\right\|<\infty$. The number $M$ is called the unconditional constant of the unconditional basis $\left(x_{n}\right)_{n=1}^{\infty}$.

Let $\left(x_{n}\right)_{n=1}^{\infty}$ be a basic sequence in $X,\left(a_{n}\right)_{n=1}^{\infty}$ be a sequence of scalars and $0 \leq k_{1}<k_{2}<\ldots$ be integers. A sequence $\left(u_{n}\right)_{n=1}^{\infty}$ of nonzero elements of $X$ of the form

$$
u_{n}=\sum_{i=k_{n}+1}^{k_{n+1}} a_{i} x_{i}
$$

is called a block basis of $\left(x_{n}\right)_{n=1}^{\infty}$. It is not hard to see that $\left(u_{n}\right)_{n=1}^{\infty}$ is a basic sequence itself, the basis constant of which does not exceed that of $\left(x_{n}\right)_{n=1}^{\infty}$. Two basic sequences $\left(x_{n}\right)_{n=1}^{\infty}$ in $X$ and $\left(y_{n}\right)_{n=1}^{\infty}$ in $Y$ are called $\lambda$-equivalent if there exists an isomorphism $T:\left[x_{n}\right] \rightarrow\left[y_{n}\right]$ between the closed linear spans of these systems with $T x_{n}=y_{n}$ for all $n$ such that $\|T\|\left\|T^{-1}\right\| \leq \lambda$. Basic sequences are said to be equivalent if they are $\lambda$-equivalent for some $\lambda \in[1,+\infty)$. Using the Closed Graph theorem, one can easily show that basic sequences $\left(x_{n}\right)_{n=1}^{\infty}$ and $\left(y_{n}\right)_{n=1}^{\infty}$ are equivalent if and only if for every sequence of scalars $\left(a_{n}\right)_{n=1}^{\infty}$ the convergence of the series $\sum_{n=1}^{\infty} a_{n} x_{n}$ and $\sum_{n=1}^{\infty} a_{n} x_{n}$ are equivalent. It is clear that if one of two $\lambda$-equivalent basic sequences is unconditional then the other one is unconditional as well, and the basic (unconditional) constants $K_{1}, K_{2}$ are estimated as follows: $\lambda^{-1} K_{1} \leq K_{2} \leq \lambda K_{1}$. 


\subsection{The Haar system in $L_{p}$}

Define the dyadic intervals by setting $I_{n}^{k}=\left[\frac{k-1}{2^{n}}, \frac{k}{2^{n}}\right)$ for $n=0,1, \ldots$ and $k=1, \ldots, 2^{n}$. The $L_{\infty}$-normalized Haar system is the following sequence in $L_{\infty}: \bar{h}_{1}=\mathbf{1}$ and

$$
\bar{h}_{2^{n}+k}=\mathbf{1}_{I_{n+1}^{2 k-1}}-\mathbf{1}_{I_{n+1}^{2 k}}
$$

for $n=0,1,2, \ldots$ and $k=1,2, \ldots, 2^{n}$ (by $\mathbf{1}_{A}$ we denote the characteristic function of a set $A$ ). The Haar system is a monotone basis of every space $L_{p}$ with $1 \leq p<\infty$ [30, p. 3], and an unconditional basis of $L_{p}$ for any $1<p<\infty$ [31, p. 155] (the first fact one can obtain using a criterium of bases, and the second fact is a deep result of Paley [36] (1932), the proof of which was then simplified by Burkholder [11] (1985)). The unconditional constant of the Haar system in $L_{p}$ equals $K_{p}=\max \{p, q\}-1$, where $1 / p+1 / q=1$ [10].

The Haar system possesses the following useful property, called the precise reproducibility [28], [31, p. 158]: for every isomorphic embedding $T: L_{p} \rightarrow X, 1 \leq p<\infty$, where $X$ is a Banach space with a basis $\left(x_{n}\right)_{n=1}^{\infty}$, and every $\varepsilon>0$ there is a block basis $\left(u_{n}\right)_{n=1}^{\infty}$ of $\left(x_{n}\right)_{n=1}^{\infty}$, which is $\left(\|T\|\left\|T^{-1}\right\|+\varepsilon\right)$-equivalent to the Haar system in $L_{p}$. This gives that the Haar system is the "best" basis: once we have an unconditional basis in $L_{p}$, the Haar system is unconditional as well, and its unconditional constant is the minimal possible one. Since the Haar system is a conditional basis in $L_{1}$ [31, p. 156], we obtain that $L_{1}$ cannot be isomorphically embedded in a Banach space with an unconditional basis (initially this was proved by A. Pełczyński [39]).

\subsection{Nonseparable $L_{p}(\mu)$-spaces}

There is a nice complete isomorphic classification of the spaces $L_{p}(\mu)$ over finite atomless measure spaces $(\Omega, \Sigma, \mu)$. A canonical representative of measure spaces $(\Omega, \Sigma, \mu)$ with $^{3}$ $\operatorname{dim} L_{p}(\mu)=\aleph_{\alpha}$ for $0<p<\infty$ is $\left(\{-1,1\}{ }^{\omega_{\alpha}}, \Sigma_{\omega_{\alpha}}, \mu_{\omega_{\alpha}}\right)$, where $\omega_{\alpha}$ is the cardinal of cardinality $\aleph_{\alpha}, \Sigma_{\omega_{\alpha}}$ is the Borel $\sigma$-algebra of subsets of $\left.\{-1,1\}\right\}^{\omega_{\alpha}}$ endowed with the Tykhonov topology on the power of the discrete two-point space $\{-1,1\}$, and $\mu_{\omega_{\alpha}}$ is the corresponding power of the measure $\mu_{0}$ on the subsets of $\{-1,1\}$ defined by $\mu_{0}\{-1\}=\mu_{0}\{1\}=1 / 2$. In other words, $\mu_{\omega_{\alpha}}$ is the Haar measure on the compact Abelian group $\{-1,1\}^{\omega_{\alpha}}$ with the point-wise product. By the famous Maharam theorem (see [32] for the original paper, and [15,23] for different proofs), every finite atomless measure space $(\Omega, \Sigma, \mu)$ is isomorphic (in the sense of measure spaces) to a unique (up to a permutation of summands) direct sum of the measure spaces $\bigoplus_{\alpha \in \mathcal{A}}\left(\{-1,1\}^{\omega_{\alpha}}, \Sigma_{\omega_{\alpha}}, \varepsilon_{\alpha} \mu_{\omega_{\alpha}}\right)$, where $\mathcal{A}$ is an at most countable set of ordinals, called the Maharam invariants of $(\Omega, \Sigma, \mu)$, and $\varepsilon_{\alpha}>0$ are weights with $\sum_{\alpha \in \mathcal{A}} \varepsilon_{\alpha}=\mu(\Omega)$. The Lebesgue measure space $([0,1], \Sigma, \lambda)$, where $\lambda$ is the Lebesgue measure on the Borel $\sigma$-algebra $\Sigma$ of subsets of $[0,1]$, is isomorphic to $\left(\{-1,1\}^{\omega_{0}}, \Sigma_{\omega_{0}}, \mu_{\omega_{0}}\right)$. As a consequence, we obtain that every $L_{p}(\mu)$-space over a finite atomless measure $\mu$ with $0<p \leq \infty$ is isometrically isomorphic to the $\ell_{p}$-sum $\left(\sum_{\alpha \in \mathcal{A}} L_{p}\{-1,1\}^{\omega_{\alpha}}\right)_{p}$.

A (not ordered) family $\left(x_{i}\right)_{i \in I}$ of elements of a (non-separable) Banach space $X$ is called an unconditional basis of $X$ if every $x \in X$ admits a unique representation $x=\sum_{i \in I} a_{i} x_{i}$, where the set of all indices $i \in I$ with $a_{i} \neq 0$ is at most countable, and the series converges unconditionally. One can show directly, that a family $\left(x_{i}\right)_{i \in I}$ with dense linear span is an unconditional basis of $X$ if and only if every its countable subfamily is an unconditional basic sequence. If this is the case then the unconditional constants of countable subfamilies are bounded from

\footnotetext{
${ }^{3}$ By $\operatorname{dim} X$ we mean the smallest cardinality of subsets of $X$ with dense linear span.
} 
above, and their supremum equals the unconditional constant of the entire family, which is defined similarly.

P. Enflo and H.P. Rosenthal (1973) [13] proved that, if $\operatorname{dim} L_{p}(\mu) \geq \aleph_{\omega_{0}}$, where $\mu$ is finite atomless and $1<p<\infty, p \neq 2$, then $L_{p}(\mu)$ does not embed isomorphically into a Banach space with an unconditional basis. They proved preliminarily that, for any $n \in \mathbb{N}$, assuming the isomorphic embedding $T: L_{p}\{-1,1\}^{\omega_{n}} \rightarrow X$ into a Banach space $X$ with an unconditional basis $\left(x_{i}\right)_{i \in I}$, the finite Walsh system $\left(w_{J}\right)_{|J| \leq n}$ is $\|T\|\left\|T^{-1}\right\|$-reproducible in $\left(x_{i}\right)_{i \in I}$, even more, $\|T\|\left\|T^{-1}\right\|$-equivalent to a suitable block basis of $\left(x_{i}\right)_{i \in I}$. As a consequence, the unconditional constant $M_{n}$ of $\left(w_{J}\right)_{|J| \leq n}$ does not exceed $M\|T\|\left\|T^{-1}\right\|$, where $M$ is the unconditional constant of $\left(x_{i}\right)_{i \in I}$. Since for every $n \in \mathbb{N}$ the space $L_{p}\{-1,1\}^{\omega_{n}}$ isometrically embeds into $L_{p}(\mu)$, it then remained to show that $M_{n} \rightarrow \infty$ as $n \rightarrow \infty$, which is true. Unfortunately, their method could not give more, remaining the following problem to be open.

Problem 5 (P. Enflo and H.P. Rosenthal, 1973, [13]). Let $1 \leq p<\infty, p \neq 2$, and let $(\Omega, \Sigma, \mu)$ be a finite atomless measure space with $\aleph_{0}<\operatorname{dim} L_{p}(\mu)<\aleph_{\omega_{0}}$. Is there an unconditional basis of $L_{p}(\mu)$ ?

Below we describe two different possible ideas to solve this problem.

\subsection{The Olevskii system}

In 1966 A.M. Olevskii constructed a system of functions on $[0,1]$, which is a basis of $L_{1}$ containing the Rademacher system as a part [35]. This system, called in the literature the Olevskii system, is a conditional basis in $L_{p}$ for $p \neq 2$, a result of E.M. Semenov [49]. If one tries to prove that $L_{p}\{-1,1\}^{\omega_{1}}$ (and therefore, $L_{p}\{-1,1\}^{\omega_{n}}$ for each $n \geq 1$ ) has no unconditional basis, then it would be enough to prove that the Olevskii system is reproducible in any unconditional basis of $L_{p}\{-1,1\}^{\omega_{1}}$. Let us present an author's description of the Olevskii system, which may be convenient for this purpose.

First, we represent the Haar system (2), collected by bunches, via the Rademacher system $\left(r_{n}\right)_{n=1}^{\infty}$ as follows:

bunch $1:$ 1,

bunch $2: r_{1}$

bunch $3: \frac{r_{1}+1}{2} \cdot r_{2}, \frac{r_{1}-1}{2} \cdot r_{2}$,

bunch $4: \frac{r_{1}+1}{2} \cdot \frac{r_{2}+1}{2} \cdot r_{3}, \frac{r_{1}+1}{2} \cdot \frac{r_{2}-1}{2} \cdot r_{3}, \frac{r_{1}-1}{2} \cdot \frac{r_{2}+1}{2} \cdot r_{3}, \frac{r_{1}-1}{2} \cdot \frac{r_{2}-1}{2} \cdot r_{3}$, $\cdots$

The Olevskii system can be constructed using the following scheme. First, we take the function 1. Then, to obtain the $(n+1)$-th Olevskii bunch, we multiply the beginning of the Haar system including its $n$-th bunch by $r_{n}$.

bunch $1: \mathbf{1}$,

bunch 2 : $r_{1}$,

bunch $3: r_{2}, r_{1} \cdot r_{2}$, 
bunch $4: r_{3}, \quad r_{1} \cdot r_{3}, \frac{r_{1}+1}{2} \cdot r_{2} \cdot r_{3}, \frac{r_{1}-1}{2} \cdot r_{2} \cdot r_{3}$,

bunch $5: \quad r_{4}, \quad r_{1} \cdot r_{4}, \frac{r_{1}+1}{2} \cdot r_{2} \cdot r_{4}, \quad \frac{r_{1}-1}{2} \cdot r_{2} \cdot r_{4}, \quad \frac{r_{1}+1}{2} \cdot \frac{r_{2}+1}{2} \cdot r_{3} \cdot r_{4}$,

$$
\frac{r_{1}+1}{2} \cdot \frac{r_{2}-1}{2} \cdot r_{3} \cdot r_{4}, \quad \frac{r_{1}-1}{2} \cdot \frac{r_{2}+1}{2} \cdot r_{3} \cdot r_{4}, \quad \frac{r_{1}-1}{2} \cdot \frac{r_{2}-1}{2} \cdot r_{3} \cdot r_{4},
$$

A partial question to this concern: is the beginning 1, $r_{1}, r_{2}, r_{1} \cdot r_{2}$ of the Olevskii system isometrically reproducible in any unconditional basis of $L_{p}\{-1,1\}{ }^{\omega_{1}}$ ? Remark that it is isometrically reproducible in any unconditional basis of $L_{p}\{-1,1\}^{\omega_{2}}$, by the Enflo-Rosenthal results, because it coincides with the Walsh system of order two.

\subsection{A close separable problem}

Consider the following important partial case of Problem 5.

Problem 6. Let $1 \leq p<\infty, p \neq 2$. Does there exist an unconditional basis in $L_{p}\{-1,1\}^{\omega_{1}}$ ?

We now pose a separable problem and then provide arguments to show that it is close to Problem 6. Let $E_{p}=L_{p}[0,1]^{2}$ be the $L_{p}$-space over the Lebesgue measure space of Borel subsets of the square $[0,1]^{2}$, and let $F_{p}$ be the subspace of $E_{p}$ consisting of all functions depending only on the first variable.

Problem 7. Let $1 \leq p<\infty, p \neq 2$. Does there exist an unconditional basis $\left(f_{n}\right) \cup\left(g_{n}\right)$ in $E_{p}$ consisting of two parts such that $\left[f_{n}\right]=F_{p}$ and the unconditional constant of $\left(f_{n}\right)$ equals the unconditional constant of the entire basis $\left(f_{n}\right) \cup\left(g_{n}\right)$ ?

Theorem 8. An affirmative answer to Problem 6 implies an affirmative answer to Problem 7.

Before the proof, we provide with some necessary information. Given an infinite set $I$, $i \in I, x \in\{-1,1\}^{I \backslash\{i\}}$, and $\theta \in\{-1,1\}$, we denote by $\theta \times x$ the element $y \in\{-1,1\}^{I}$ such that $y(i)=\theta$ and $y(j)=x(j)$ for all $j \in I \backslash\{i\}$. Following [13], a $\mu_{I}$-measurable function $f:\{-1,1\}^{I} \rightarrow \mathbb{R}$ is said to be independent of $i \in I$, if $f(1 \times x)=f(-1 \times x)$ for $\mu_{I \backslash\{i\}^{-}}$ almost all values of $x \in\{-1,1\}^{I \backslash\{i\}}$. In the opposite case we say that $f$ depends on $i$. For any measurable function $f:\{-1,1\}^{I} \rightarrow \mathbb{R}$, the set $\{i \in I: f$ depends on $i\}$ is at most countable. By the obvious reason, the same terminology we apply to equivalence classes of measurable functions.

Proof. Let $\left(f_{\alpha}\right)_{\alpha<\omega_{1}}$ be an unconditional basis of $L_{p}\{-1,1\}^{\omega_{1}}$ with unconditional constant $M$. For any $\alpha<\omega_{1}$ we denote by $X_{\alpha}$ the subspace of all $f \in L_{p}\{-1,1\}^{\omega_{1}}$ depending on coordinates $<\alpha$ only. Obviously, $X_{\alpha}$ is isometrically isomorphic to $L_{p}\{-1,1\}^{\alpha}$, which is separable and atomless, and hence, isometrically isomorphic to $L_{p}$.

Lemma 1. There exists a strictly increasing $\omega_{1}$-sequence of limited ordinals $\left(\xi_{\gamma}\right)_{\gamma<\omega_{1}}, \xi_{\gamma}<\omega_{1}$, such that $\left[f_{\alpha}\right]_{\alpha<\xi_{\gamma}}=X_{\xi_{\gamma}}$ for all $\gamma<\omega_{1}$.

Proof of Lemma 1. Since every function $f \in L_{p}\{-1,1\}^{\omega_{1}}$ depends on at most countable set of ordinals $\alpha<\omega_{1}$, for every separable subspace $Z$ of $L_{p}\{-1,1\}^{\omega_{1}}$ the value $\varphi(Z)=\min \{\alpha<$ $\left.\omega_{1}: Z \subseteq X_{\alpha}\right\}$ is well defined. Then

$$
Z \subseteq X_{\varphi(Z)} \text { for every separable subspace } Z \text {. }
$$


Since every function $f \in L_{p}\{-1,1\}^{\omega_{1}}$ has an expansion $f=\sum_{\alpha<\omega_{1}} a_{\alpha} f_{\alpha}$, where the set $\left\{\alpha<\omega_{1}: a_{\alpha} \neq 0\right\}$ is at most countable, for every separable subspace $Z$ of $L_{p}\{-1,1\}^{\omega_{1}}$ the value $\psi(Z)=\min \left\{\beta<\omega_{1}: Z \subseteq\left[f_{\alpha}\right]_{\alpha<\beta}\right\}$ is well defined as well. Then

$$
Z \subseteq\left[f_{\alpha}\right]_{\alpha<\psi(Z)} \text { for every separable subspace } Z \text {. }
$$

Now define recursively $\omega_{1}$-sequences $\left(\alpha_{\eta}\right)_{\eta<\omega_{1}}$ and $\left(\beta_{\eta}\right)_{\eta<\omega_{1}}$ possessing the following properties for every $\eta<\zeta<\omega_{1}$ :

1. $\alpha_{\eta} \leq \beta_{\eta}<\alpha_{\zeta}$;

2. $\left[f_{\alpha}\right]_{\alpha<\alpha_{\eta}} \subseteq X_{\beta_{\eta}} \subseteq\left[f_{\alpha}\right]_{\alpha<\alpha_{\zeta}}$.

Set $\alpha_{0}=\omega_{0}$ and $\beta_{0}=\max \left\{\varphi\left(\left[f_{\alpha}\right]_{\alpha<\alpha_{0}}\right), \omega_{0}\right\}$. Then $\alpha_{0} \leq \beta_{0}$ and $\left[f_{\alpha}\right]_{\alpha<\alpha_{0}} \subseteq X_{\beta_{0}}$. Given any $\delta<\omega_{1}$, we assume that $\delta$-sequences $\left(\alpha_{\eta}\right)_{\eta<\delta}$ and $\left(\beta_{\eta}\right)_{\eta<\delta}$ possessing 1 and 2 for every $\eta<\zeta<\delta$ have been already constructed. To define $\alpha_{\delta}$ and $\beta_{\delta}$, we consider cases.

(i) $\delta$ is an isolated ordinal, that is, $\delta=\delta^{\prime}+1$. In this case we set

$$
\alpha_{\delta}=\max \left\{\psi\left(X_{\beta_{\delta^{\prime}}}\right), \beta_{\delta^{\prime}}+1\right\} \text { and } \beta_{\delta}=\max \left\{\varphi\left(\left[f_{\alpha}\right]_{\alpha<\alpha_{\delta}}\right), \alpha_{\delta}\right\} \text {. }
$$

(ii) $\delta$ is a limited ordinal. In this case we set

$$
\alpha_{\delta}=\beta_{\delta}=\bigcup_{\eta<\delta} \alpha_{\eta}=\bigcup_{\eta<\delta} \beta_{\eta}
$$

(the latter equality is guaranteed by property 1 for every $\eta<\zeta<\delta$ ).

Property 1 for $\eta<\zeta \leq \delta$ follows directly from the construction. To prove 2, observe

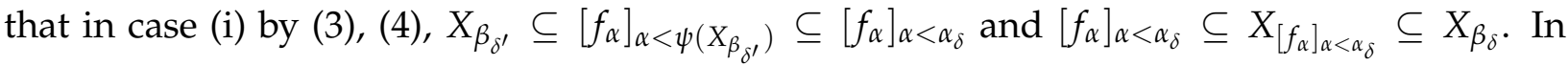
case (ii) inclusions 2 are obvious. Thus, the desired $\omega_{1}$-sequences $\left(\alpha_{\eta}\right)_{\eta<\omega_{1}}$ and $\left(\beta_{\eta}\right)_{\eta<\omega_{1}}$ are constructed.

By (ii) and 2, for every limited ordinal $\delta<\omega_{1}$ one has $\left[f_{\alpha}\right]_{\alpha<\alpha_{\delta}}=X_{\alpha_{\delta}}$. By (ii), for every limited ordinal $\delta<\omega_{1}$, the ordinal $\alpha_{\delta}$ is limited as well. Since there are uncountably many such ordinals, we can renumber them to obtain the desired $\omega_{1}$-sequence.

Lemma 2. Let $I \subset J$ be countable subsets with $J \backslash I$ infinite. Then there is an isometric isomorphism $T: E_{p} \rightarrow L_{p}\{-1,1\}^{J}$ such that $T\left(F_{p}\right)$ equals the subspace of $L_{p}\{-1,1\}^{J}$ consisting of all functions which depend on coordinates $i \in I$ only.

Proof of Lemma 2. It is straightforward that the linear span of the Walsh system $\left(w_{A}\right)_{A \in \mathbb{N}<\omega}$ coincides with that of the Haar system, hence it is dense in $L_{p}$. So, to define an isometrical isomorphism on the entire $L_{p}(\mu)$, it is enough to define it on the Walsh system and prove that it is an isometry on the linear span. Observe that the Walsh system in $E_{p}=L_{p}[0,1]^{2}$ is given by $w_{A}(x) w_{B}(y)$, where $A, B$ are finite subsets of $\mathbb{N}$.

Let $I=\left\{i_{1}, i_{2}, \ldots\right\}$ and $J \backslash I=\left\{j_{1}, j_{2}, \ldots\right\}$ be any numerations. Given any $A, B \in \mathbb{N}^{<\omega}$, we define functions $\widehat{w}_{A}^{\prime}, \widehat{w}_{B}^{\prime \prime}:\{-1,1\}^{J} \rightarrow \mathbb{R}$ by setting $\widehat{w}_{A}^{\prime}(x)=\prod_{n \in A} x\left(i_{n}\right)$ and $\widehat{w}_{B}^{\prime \prime}(x)=$ $\prod_{n \in B} x\left(j_{n}\right)$. Likewise, the Walsh system in $L_{p}\{-1,1\}^{J}$ can be represented as follows: $\widehat{w}_{A}^{\prime} \cdot \widehat{w}_{B}^{\prime \prime}, A, B \in \mathbb{N}^{<\omega}$. Now we define $T: E_{p} \rightarrow L_{p}\{-1,1\}^{J}$, first on the Walsh system by $T w_{A}(x) w_{B}(y)=\widehat{w}_{A}^{\prime} \cdot \widehat{w}_{B}^{\prime \prime}$ for all $A, B \in \mathbb{N}^{<\omega}$, and then extend to the linear span of the Walsh system $W$ by linearity. We omit a routine proof that the obtained mapping is an isometry on $W$. It remains to observe that $T\left(F_{p}\right)=L_{p}\{-1,1\}^{I}$. 
We continue the proof of the theorem. Take a sequence $\left(\xi_{\gamma}\right)_{\gamma<\omega_{1}}$ satisfying the claims of Lemma 1. Denote by $M_{\gamma}$ the unconditional constant of the system $\left(f_{\alpha}\right)_{\alpha<\xi_{\gamma}}$. Then $M_{\gamma} \uparrow M$. Since there is no strictly increasing $\omega_{1}$-sequence of reals, we obtain that there is $\gamma_{0}<\omega_{1}$ such that $M_{\gamma}=M$ for all $\gamma_{0} \leq \gamma<\omega_{1}$. Choose by Lemma 2 an isometric isomorphism $T: E_{p} \rightarrow X_{\xi_{\gamma_{0}+1}}$ with $T\left(F_{p}\right)=X_{\xi_{\gamma_{0}}}$. Since $\left(f_{\alpha}\right)_{\alpha<\xi_{\gamma_{0}+1}}=\left(f_{\alpha}\right)_{\alpha<\xi_{\gamma_{0}}} \cup\left(f_{\alpha}\right)_{\xi_{\gamma_{0}} \leq \alpha<\xi_{\gamma_{0}+1}}$ is an unconditional basis of $X_{\xi_{\gamma_{0}+1}}$ with unconditional constant $M$ and $\left(f_{\alpha}\right)_{\alpha<\xi_{\gamma_{0}}}$ is an unconditional basis of $X_{\xi_{\gamma_{0}}}$ with with the same unconditional constant $M$, we obtain that $\left(T^{-1} f_{\alpha}\right)_{\alpha<\xi_{\gamma_{0}+1}}=$ $\left(T^{-1} f_{\alpha}\right)_{\alpha<\xi_{\gamma_{0}}} \cup\left(T^{-1} f_{\alpha}\right)_{\xi_{\gamma_{0}} \leq \alpha<\xi_{\gamma_{0}+1}}$ is an unconditional basis of $T^{-1}\left(X_{\xi_{\gamma_{0}+1}}\right)=E_{p}$ with unconditional constant $M$ and $\left(T^{-1} f_{\alpha}\right)_{\alpha<\xi_{\gamma_{0}}}$ is an unconditional basis of $T^{-1}\left(X_{\xi_{\gamma_{0}}}\right)=F_{p}$ with with the same unconditional constant $M$.

\section{Remarks.}

1. In Problem 7, one can equivalently replace the unconditional constants of unconditional bases with the supremum of norms of projections with respect to the bases.

2. We do not know of whether an affirmative solution to Problem 7 formally implies an affirmative solution to Problem 6, however, an affirmative solution to Problem 7 would give a possible way to construct an unconditional basis of $L_{p}\{-1,1\}^{\omega_{1}}$ by a recursive procedure.

\section{REFERENCES}

[1] Albiac F., Kalton N. Topics in Banach Space Theory. Graduate Texts in Math., 233, Springer, New York, 2006.

[2] Alspach D., Enflo P., Odell E. On the structure of separable $\mathcal{L}_{p}$ spaces $(1<p<\infty)$. Stud. Math. 1977, 60, 79-90.

[3] Alspach D., Odell E. $L_{p}$ spaces. Handbook of the geometry of Banach spaces. Vol. I. Eds.: W.B. Johnson, J. Lindenstrauss. North-Holland Publishing Co., Amsterdam, 2001, 123-159.

[4] Banach S.S. Course of Functional Analysis. Rad. Shkola, Kyiv, 1948 (in Ukrainian). Translation from the book “Théorie des opérations linéaires", 1932.

[5] Bennet B., Dor L.E., Goodman V., Johnson W.B., Newman C.M. On uncomplemented subspaces of $L_{p}, 1<p<2$. Israel J. Math. 1977, 26 (2), 178-187.

[6] Bourgain J. A counterexample to a complementation problem. Compos. Math. 1981, 43 (1), 133-144.

[7] Bourgain J. A characterization of non-Dunford-Pettis operators on L $L^{1}$. Israel J. Math. 1980, 37 (1-2), 48-53.

[8] Bourgain J., Rosenthal H.P., Schechtman G. An ordinal $L^{p}$-index for Banach spaces, with applications to complemented subspaces of $L^{p}$. Ann. of Math. 1981, 114 (2), 193-228. doi:10.2307/1971293

[9] Bretagnolle J., Dacunha-Castelle D., Krivine J.L. Lois stables et espaces $L_{p}$. Ann. Inst. Henri Poincare, Sér. B. 1966, 2, 231-259.

[10] Burkholder D.L. A nonlinear partial differential equation and the unconditional constant of the Haar system in $L^{p}$. Bull. Amer. Math. Soc. (N.S.) 1982, 7 (3), 591-595.

[11] Burkholder D.L. An elementary proof of an inequality of R. E. A. C. Paley. Bull. Lond. Math. Soc. 1985, 17 (5), 474-478.

[12] Diestel J., Uhl J.J. Vector Measures. AMS, Mathematical surveys, 1977.

[13] Enflo P., Rosenthal H.P. Some results concerning $L^{p}(\mu)$-spaces. J. Funct. Anal. 1973, 14 (4), 325-348.

[14] Enflo P., Starbird T. Subspaces of $L^{1}$ containing $L^{1}$. Studia Math. 1979, 65 (2), 203-225.

[15] Fremlin D.H., Measure Theory. Vol. 3. Measure algebras. Torres Fremlin, Colchester, 2004. 
[16] Grothendieck A. Produits tensoriels topologiques et espaces nucléaires. Mem. Amer. Math. Soc. $1955,16$.

[17] Hagler J. A counterexample to several questions about Banach spaces. Studia Math. 1977, 60, 289-308.

[18] Johnson W.B., Maurey B., Schechtman G., Tzafriri L. Symmetric structures in Banach spaces. Mem. Amer. Math. Soc. 1979, 217.

[19] Johnson W.B., Odell E. Subspaces of $L_{p}$ which embed into $\ell_{p}$. Compos. Math. 1974, 28 (1), 37-49.

[20] Kadec M.I. On the linear dimension of the spaces $L_{p}$ and $\ell_{q}$. Uspekhi Mat. Nauk. 1958, 13 (6), 95-98. (in Russian)

[21] Kadec M.I., Pełczyński A. Bases, lacunary sequences and complemented subspaces in the spaces $L_{p}$. Studia Math. 1962, 21 (2), 161-176.

[22] Kalton N.J. Isomorphisms between $L_{p}$-function spaces when $p<1$. J. Funct. Anal. 1981, 42 (3), $299-337$.

[23] Lacey H.E. The Isometrical Theory of Classical Banach Spaces. Springer-Verlag, Berlin-Heidelberg-New York, 1974.

[24] Lévy M. Prolongement d'un opérateur d'un sous-espace de $L^{1}(\mu)$ dans $L^{1}(v)$. Séminaire d'Analyse Fonctionnelle 1979-1980. Exposé 5. Ecole Polytechnique, Palaiseau, 1980.

[25] Levy P. Theorie de l'addition de variable aleatoires. Gauthier-Villars, Paris, 1937.

[26] Lewis D.R., Stegall C. Banach spaces whose duals are isomorphic to $\ell_{1}(\Gamma)$. J. Funct. Anal. 1973, 12, $177-187$.

[27] Lindenstrauss J., Pełczyński A. Absolutely summing operators in $L_{p}$-spaces and their applications. Studia Math. 1968, 29 (3), 275-326.

[28] Lindenstrauss J., Pełczyński A. Contribution to the theory of classical Banach spaces. J. Funct. Anal. 1971, 8 (2), 225-249.

[29] Lindenstrauss J., Rosenthal H.P. The $\mathcal{L}_{p}$ spaces. Israel J. Math. 1969, 7 (4), 325-349.

[30] Lindenstrauss J., Tzafriri L. Classical Banach spaces, Vol. 1, Sequence spaces. Springer-Verlag, BerlinHeidelberg-New York, 1977.

[31] Lindenstrauss J., Tzafriri L. Classical Banach spaces, Vol. 2, Function spaces. Springer-Verlag, BerlinHeidelberg-New York, 1979.

[32] Maharam D. On homogeneous measure algebras. Proc. Natl. Acad. Sci. USA 1942, 28, 108-111.

[33] Maslyuchenko O.V., Mykhaylyuk V.V., Popov M.M., A lattice approach to narrow operators, Positivity 2009, 13, 459-495.

[34] Maurey B. Sous-espaces compléméntes de L d'apres P. Enflo. Semin. Maurey-Schwartz 1974-75, III, 1-14.

[35] Olevskii A.M. An orthonormal system and its applications. Math. Sb. (N.S.) 1966, 71 (113), 297-336. (in Russian)

[36] Paley R.E.A.C. A remarkable series of orthogonal functions. I. Proc. Lond. Math. Soc. 1932, 34, $241-264$.

[37] Paley R.E.A.C. Some theorems on abstract spaces. Bull. Amer. Math. Soc. 1936, 42 (4), 235-240.

[38] Pełczyński A. Projections in certain Banach spaces. Studia Math. 1960, 19 (3), 209-228.

[39] Pełczyński A. On the impossibility of embedding of the space L in certain Banach spaces. Colloq. Math. 1961, 8, 199-203.

[40] Pisier G. Complex interpolation and regular operators between Banach lattices. Arch. Math. (Basel) 1994, 62, 261269.

[41] Popov M., Randrianantoanina B. Narrow Operators on Function Spaces and Vector Lattices. De Gruyter Studies in Mathematics 45, De Gruyter, Berlin-Boston, 2013.

[42] Rosenthal H.P. Projections onto translation-invariant subspaces of $L^{p}(G)$. Mem. Amer. Math. Soc. $1966,63$.

[43] Rosenthal H.P. On subspaces of $L^{p}(p>2)$ spanned by sequences of independent random variables. Israel J. Math. 1970, 8 (3), 273-303.

[44] Rosenthal H.P. On subspaces of $L^{p}$. Ann. of Math. 1973, 97 (2), 344-373. 
[45] Rosenthal H.P. Convolution by a biased coin. Altgeld Book (Univ. of Illinois) 1975-76, II.

[46] Rosenthal H.P. On an inequality of A. Grothendieck concerning operators on $L^{p}$. Positivity 1998, 2, 115-122.

[47] Schaefer H.H. Banach Lattices and Positive Operators. Springer-Verlag. Berlin-Heidelberg-New York, 1974.

[48] Schechtman G. Examples of $\mathcal{L}_{p \text {-spaces }}(1<p \neq 2<\infty)$. Israel J. Math. 1975, 22 (2), 138-147.

[49] Semenov E.M. Basis properties of the Olevskii system. Math. Notes 1993, 54 (3-4), 977-978. doi: 10.1007/BF01209565 (translation of Mat. Zametki 1993, 54 (3), 155-156. (in Russian))

Received 30.11.2019

Попов М.М. Про дві давні нерозв'язані проблеми про простори L $L_{p} / /$ Карпатські матем. публ. 2020. — Т.12, №1. — С. 229-241.

Аану замітку написано при підготовці доповіді на міжнародній конференції, присвяченій 70-річчю професора О. Аопушанського, 16-19 вересня 2019 р. Ми зосереджуємося на двох давніх відкритих проблемах. Перша, що належить $\Lambda$ інденштраусу і Розенталю (1969р.), формулюється так: чи кожний доповнювальний нескінченновимірний підпростір простору $L_{1}$ ізоморфний до $L_{1}$ чи до $\ell_{1}$ ? $\Delta$ руга проблема була поставлена Енфло і Розенталем у 1973 р.: чи існує несепарабельний простір $L_{p}(\mu)$ зі скінченною безатомною мірою $\mu$ та $1<p<\infty, p \neq 2$, з безумовним базисом? У замітці наведено аналіз часткових результатів та природних ідей розв'язання даних проблем.

Ключові слова і фрази: простори $L_{p}$, доповнювальний підпростір, безумовний базис. 